FERMILAB-Conf-04/147-AD August 2004

\title{
Computational Accelerator Physics Working Group Summary
}

\author{
John R. Cary*广 and Courtlandt L. Bohn ${ }^{* * \dagger}$ \\ ${ }^{*}$ University of Colorado, Boulder, CO 80309 \\ $\dagger$ also Tech-X Corporation, 5621 Arapaho Ave., Suite A, Boulder, CO 80303 \\ ${ }^{* *}$ Northern Illinois University, DeKalb, IL 60115 \\ "also Fermilab, P. O. Box 500, Batavia, IL 60510-0500
}

\begin{abstract}
.
The working group on computational accelerator physics at the $11^{\text {th }}$ Advanced Accelerator Concepts Workshop held a series of meetings during the Workshop. Verification, i.e., showing that a computational application correctly solves the assumed model, and validation, i.e., showing that the model correctly describes the modeled system, were discussed for a number of systems. In particular, the predictions of the massively parallel codes, OSIRIS and VORPAL, used for modeling advanced accelerator concepts, were compared and shown to agree, thereby establishing some verification of both codes. In addition, a number of talks on the status and frontiers of computational accelerator physics were presented, to include the modeling of ultrahigh-brightness electron photoinjectors and the physics of beam halo production. Finally, talks discussing computational needs were presented.
\end{abstract}

\section{INTRODUCTION}

This is the second time that a special group on computational accelerator physics has been a part of the Advanced Accelerator Concepts Workshop. Attention to computational accelerator physics is appropriate, as it has become increasingly useful to the accelerator physics community. As noted in one working group session, we do computation to discover new physics, design new devices, and check analytical theory and its approximations. For these purposes we must ensure that we faithfully model the basic physics. In addition, we use computation for failure analysis and optimization of existing systems and devices, and we use computation to help understand what is going on in experiments. For these purposes, we must not only get right some basic physics, we must also ensure that our computations include all of the physics needed to faithfully model experiments.

Computation is a rapidly growing methodology for studying accelerator systems, and it has become increasingly technical, requiring computational researchers to have knowledge of the message passing interface for massively parallel computing, visualization, graphical user interfaces, etc. In addition, to assure both themselves and others of the value of their computations, computational physicists must increasingly adhere to software engineering principles and methodologies. Finally, as computational resources are always limited, computationalists are continually developing new methods for solving larger problems on existing hardware. 
Talks oriented towards all of these areas were presented as part of this working group. Of course, there was significant overlap, but in some broad classification, contributed talks were in the areas of validation and verification of electromagnetic particle-incell codes [1], reduced models for modeling advanced accelerator topics [2, 3, 4], laser interactions with solid density materials [5, 6], space charge modeling [7, 8, 9], laser wake field acceleration (LWFA) [10, 11, 12, 13], plasma wake field accelerators (PWFA) [14, 15, 16], and other computational talks [17, 18, 19]. In addition, plenary talks on advances in simulation capability [20], PIC simulations of high-energy particle generation [21], halo modeling [22], and injector modeling [23] were presented.

As part of the charge to the working group was "code benchmarking," the working group had presentations on Verification and Validation, as this is more precisely known. Several talks not originally specific to this topic did additionally address it. The areas of electromagnetic particle-in-cell (EM-PIC), injector modeling, and ionization in laserplasma interactions were discussed.

What follows is a summary of the deliberations written within the perspectives of their respective contexts. The focus is on verification and validation of codes. However, as is also summarized, the deliberations went beyond that focus and into the identification of frontiers in computational physics related to advanced accelerator concepts. The concluding section lists specific areas that are ripe for research in this regard.

\section{VERIFICATION AND VALIDATION}

A charge to the working group was to "put forward a plan for benchmarking codes of the same type against each other, and well as codes of different types against each other to the extent possible." The working group accepted this charge within the modern concepts of Verification and Validation. Computational applications have the goal of solving some model of a physical system. For example, a model for LWFA is that electrons interact classically with an electromagnetic field. Verification is a process of determining whether the model is being correctly solved - thus showing that the algorithms, methodologies, and numerical parameter choices are valid. On the other hand, in the experiment being modeled, there may be residual neutrals from incomplete ionization, and continued ionization may deplete the energy of the laser pulse. A standard EM-PIC code without neutrals or ionization will not capture this effect. Validation is the process of assuring that the code has all of the needed physics to properly model the experiment.

\section{Verification of EM-PIC codes for advanced accelerator modeling}

One exercise undertaken by this working group was the verification of EM-PIC codes. Verification of these codes was, of course, previously done through comparison with exactly solvable problems, such as linear modes. To verify a code in regimes where there are no exact analytic solutions, such as strongly nonlinear, multidimensional situations, the only methodology is to compare the output of two codes that are solving 
the same problem. Thus, Fonseca [1] led such an effort, including setting up a web site http://zamb.ist.utl.pt/aac/. At that web site are listed multiple code candidates for comparisons. All code developers were invited to participate. Ultimately, the developers of the OSIRIS [24] and VORPAL [25] codes participated in the code comparisons.

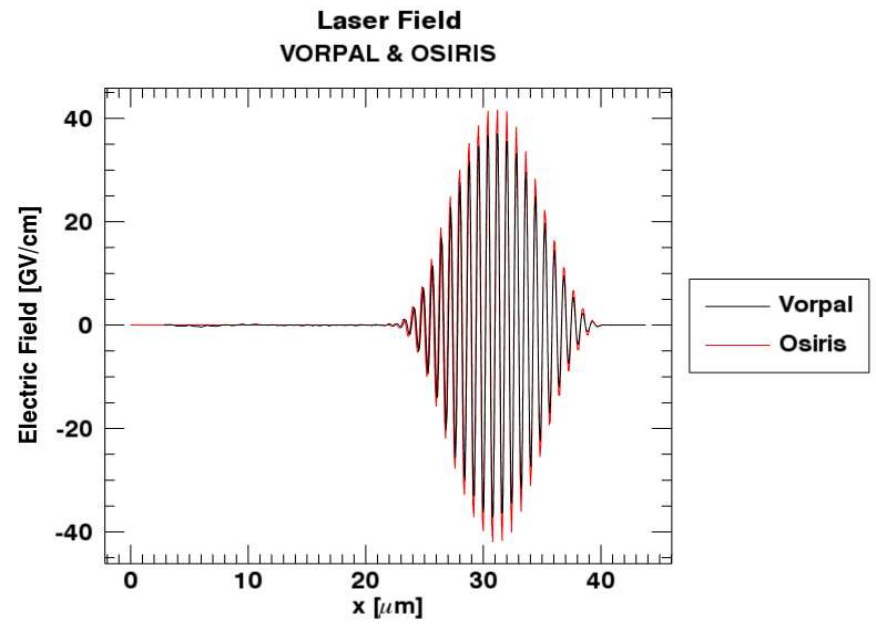

FIGURE 1. The laser electric field for an intense pulse propagated into a plasma as computed by both OSIRIS and VORPAL.

The initial problem to be solved is the wake field induced by a strong laser pulse propagating into a plasma. One result of that work was a comparison of the central lineouts for the laser electric field and the longitudinal field of the wake. The on-axis laser pulse electric field is shown in Fig. 1 as computed by both codes. One can see that the field is slightly larger in Osiris.

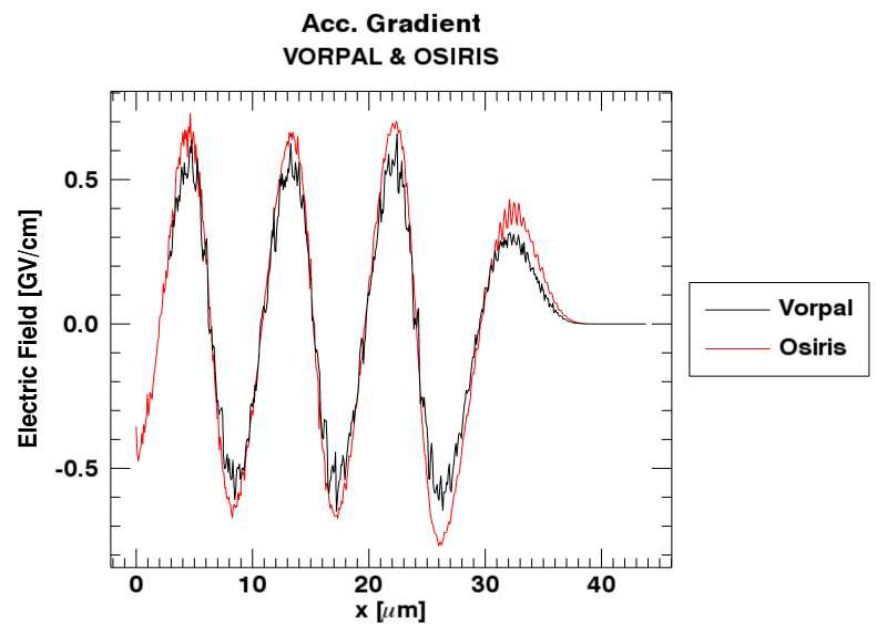

FIGURE 2. The laser electric field for an intense pulse propagated into a plasma as computed by both OSIRIS and VORPAL.

The longitudinal field is shown in Fig. 2. This comparison shows that the wake field computed by Vorpal is smaller, but in being so consistent with the smaller laser 
pulse. The VORPAL computed longitudinal field is also noisier. These differences were pursued and understood at the Workshop. In the VORPAL setup, the pulse was propagated a longer distance, thus, it had diffracted more by the time of measurement. In addition, VORPAL had no smoothing turned on, while OSIRIS did. Fonseca and the working group concluded that elimination of these differences in problem setup would lead to agreement, and so further work was deferred until after the workshop.

It was noted that carrying out such comparisons is a tedious process. One must get every last detail correct. For the above case, a partial list of the differences between the two runs and codes follows:

- Laser parameters

- Temporal profile

- Transverse profile parameter definitions

- Launch methods

- Plasma parameters (including shape and length of ramp up from vacuum)

- Boundary conditions (e.g., periodic, conducting, or wave absorbing)

- Particle loading (e.g., regular grid, bit reversed, or random) affects noise

Removing some differences required modification of code capabilities. For example, a particular pulse profile might be implemented in one code but not the other.

\section{Verification and validation of electron-injector codes}

To open the discussion on verification/validation of injector codes, Court Bohn summarized a recent attempt by Daniel Mihalcea to compare code results in the context of the flat-beam experiment being conducted at the Fermilab/NICADD Photoinjector Laboratory [26]. Generating a flat beam involves imparting a nonzero angular momentum to the beam by way of a nonzero magnetic field at the cathode surface, yielding what is called a magnetized beam, and then transforming this angular momentum into an asymmetric phase space by way of a series of skew-quadrupole magnets. The idea is to achieve a large ratio between the root-mean-square emittances $\varepsilon_{y}$ and $\varepsilon_{x}$ corresponding to the transverse axes $y$ and $x$, respectively. Table 1 provides measured data from an example experiment. It also provides computed data using the codes ASTRA and PARMELA3D, the latter without and with space charge (SC) activated. The simulations incorporate an assumed transverse laser profile at the cathode. As the Table indicates, even were the measurement uncertainty in the bunch charge large, both codes agree reasonably well with the measured value of the large emittance. However, they markedly disagree with the measured small emittance, and in turn with the emittance ratio. The explanation for the discrepancy lies in the assumed laser profile. If the profile is irregular and time-varying, as it is often observed to be, computations indicate the rms properties of the beam can strongly differ from those associated with a uniform profile. Thus, for example, a laser profile that features a hole will yield a considerably larger emittance, e.g., in keeping with the measured emittance in Table 1. Accordingly, this example of code verification/validation reflects the interplay between experiment and simulation. 
TABLE 1. Example: Verification of injector codes

\begin{tabular}{lrllr}
\hline & $Q(\mathbf{n C})$ & $\varepsilon_{x}$ & $\varepsilon_{y}$ & $\varepsilon_{y} / \varepsilon_{x}$ \\
\hline Experiment & 0.6 & 1.68 & 27.71 & 16.5 \\
ASTRA & 0.6 & 0.48 & 27.28 & 56.8 \\
PARMELA3D (no SC) & 0.6 & 0.50 & 28.60 & 57.2 \\
PARMELA3D (with SC) & 0.6 & 0.51 & 28.64 & 56.2 \\
PARMELA3D (no SC) & 1.0 & 1.11 & 28.89 & 26.0 \\
PARMELA3D (with SC) & 1.0 & 1.13 & 28.99 & 25.7 \\
\hline
\end{tabular}

An important point concerning the flat-beam experiment is that the standard approach to emittance compensation becomes invalid once a nonzero magnetic field is applied to the cathode. The measured geometric rms emittance obtained for the magnetized beam is consistently larger than that obtained for the standard, unmagnetized beam. The emittance-compensation procedure must accordingly be modified for magnetized beams. Motivated by electron-cooling requirements for RHIC, Xiangjun Chang has been working on a recipe for emittance compensation of magnetized beams that are strongly dominated by space charge, and he presented his findings. His recipe, which also includes chromaticity effects, is a generalization of the standard technique for unmagnetized beams. It involves a trade-off between solenoid strength, the drift distance between the gun and first linac cavity, and the longitudinal drive-laser profile to minimize the beam emittance; for electron cooling in RHIC, Chang believes the final normalized rms emittance can be less than $55 \mu \mathrm{m}$.

Working-group discussions concerning beam production and halo formation served to highlight the critical importance of initial conditions. Irving Haber pointed out that in comparisons of simulations of the electron gun at the University of Maryland Electron Ring (UMER) with measured beam properties, the code results likewise are sensitive to the assumed initial conditions. This applies not only to rms properties, but also to halo production. In his plenary talk, Rami Kishek likewise pointed to the interplay of simulations versus experiment leading to the discovery that small quadrupole rotation errors can lead to copious halo. And in his plenary talk, John Lewellen emphasized the need for a code that accurately accounts for the evolving hierarchies of spatial and temporal scales. The moral of all these discussions and findings is that details do matter; details such as proper cathode (beam-formation) physics, alignment and field errors, and space-charge fluctuations all collaborate toward establishing even the gross beam properties. In turn, basic R\&D concerning beam physics must likewise continue.

\section{Validation of ionization physics in EM-PIC codes}

Increasingly, ionization effects have been found to be important in advanced acceleration concepts. Consequently, this physics is being added to our codes. However, now a question of validation comes up. Is our model for ionization (e.g., the use of the ADK ionization rate for field ionization) correct? Do we have correct values for the gas density? Answering these questions requires that we go through a validation exercise, where 
comparisons between code results and experimental results are made.

Bruhwiler [15] made one such comparison. The ionization of a laser pulse propagating through a neutral gas causes a blue shift of the laser light. Bruhwiler compared, in Fig. 3, the results computed in his simulation with experimental results. With the observed good agreement, one can now have confidence in other calculations that use the associated ionization package (IONPACK) that was used in these simulations.

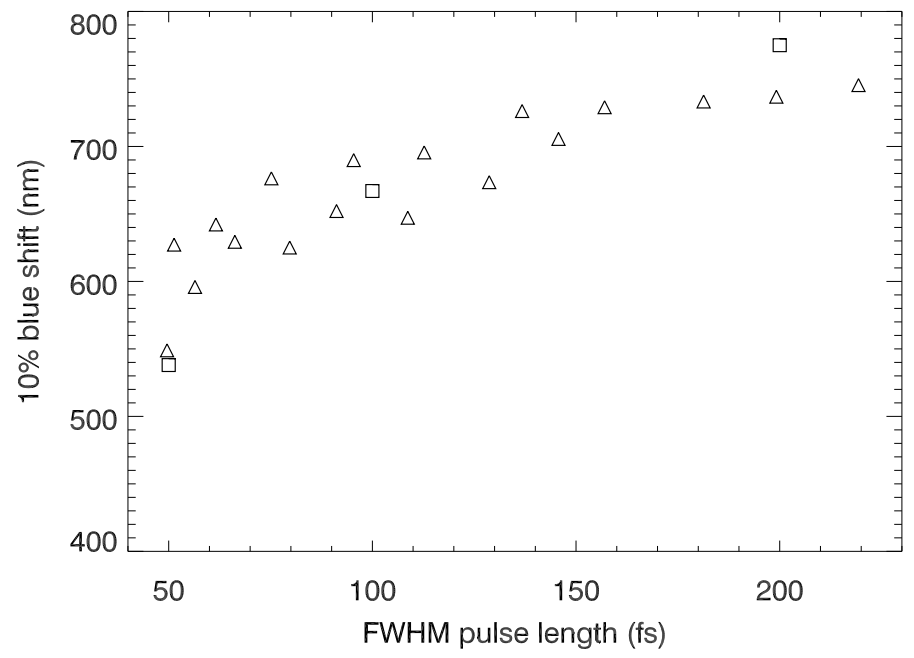

FIGURE 3. Comparison of ionization induced blue shift as computed by OOPIC PRO using the IONPACK ionization library with the experimental results. The squares are computed values, while the triangles are experimental values.

\section{Consequences of verification}

Verification gives us confidence that we are solving the assumed physics correctly. Thus, the predictions can be assumed correct to the degree that the experiment has been designed to have only that physics. Many of the talks presented at the workshop were in this context. For example, Bohn [7] deduced that oscillations and colored noise can cause dramatic growth of beam halos. Kesar [17] showed that the emission per unit length from short Smith-Purcell radiation generators can be four times larger than for very long systems. Shadwick [3] pointed out that for typical plasma temperatures, pressure effects do not significantly change the laser generated wake field. Rosenzweig [14] showed that the inverse-square scaling of the peak accelerating field with pulse length in PWFA breaks down for sufficiently short pulse lengths. In each of these cases, the codes had been verified, and so predictions about idealized situations are possible.

\section{Validation needs}

At present, validation is a cottage industry, with each act of validation isolated. Moreover, it can be difficult, as it requires a close interaction with experimentalists, and for many computationalists in our community, the appropriate experiments are 
not geographically close. To improve upon this situation, the working group proposed that experimentalists provide well diagnosed, standard experiments rich in physics. The parameters should be available for computationalists to compare with.

\section{FRONTIERS IN COMPUTING}

Computing continues to advance through the introduction of new algorithms and physics into our computational applications. One area of algorithmic need is in modeling the LWFA, for which brute force methods break down upon consideration of the full system due to the extremely large scale separations in LWFA modeling. The smallest scale in such models is the laser wavelength, of the order of $1 \mu \mathrm{m}$. On the other hand, an LWFA $\mathrm{GeV}$ stage would likely be 10's of cm long. A collection of stages for a TeV collider would be tens of meters long. Therefore, direct numerical simulation would have to span a range of $10^{7}$ in spatial scales. Another area is in ionization physics, critical to advanced acceleration concepts. This requires the development of libraries for use by the community.

Three talks were presented on the reduction of scale separation. Hur [2] has been developing an averaged PIC method. This method averages over the laser oscillations to remove the laser wavelength and frequency from the problem. A more severe approximation, with greater elimination of scale separation was presented in the work on photon kinetics by Reitsma. In this work the photons are tracked on the focusing time, while the particles are tracked on the betatron period. This work is currently limited to 1D.

Another code that reduces the problem to the same time scales is QuickPIC, which was discussed by Cooley [4]. The work of Cooley et al is very promising, as it reduces the scale separation while also allowing for 2D computations. Moreover, Cooley et al have also undertaken verification studies that show good agreement. Comparisons of QuickPIC with direct numerical simulation are shown in Fig. 4. These results show that the original, base QuickPIC algorithm did not give sufficient agreement (the verification was unsuccessful). However, further work yielded the full QuickPIC algorithm, which was verified against full PIC simulations.

A second major frontier is in the inclusion of ionization effects in our advanced accelerator codes. The validation of such computations was discussed earlier. The importance of this work was demonstrated by Bruhwiler et al [15], who have shown that field ionization by the head of a beam pulse can create the plasma necessary for the afterburner to succeed under certain conditions. This work was done with the XOOPIC simulation code. Now, field and impact ionization are making their way into the OSIRIS and VORPAL codes.

To assist in this effort, Dimitrov et al [16] have been developing the IONPACK library, available at http://www.txcorp.com/technologies/IONPACK/. This library is written in $\mathrm{C}$ so as to make it callable from Fortran90, Fortran77, C++, and Java. It is cross platform, maintained on Linux, Mac OS X, Windows, and AIX. The hope is that with this library available, it will no longer be necessary for each computationalist to develop code or ionization calculations. This library is used in the VORPAL and OopicPro codes, and it is a certified module for the LSP code. 


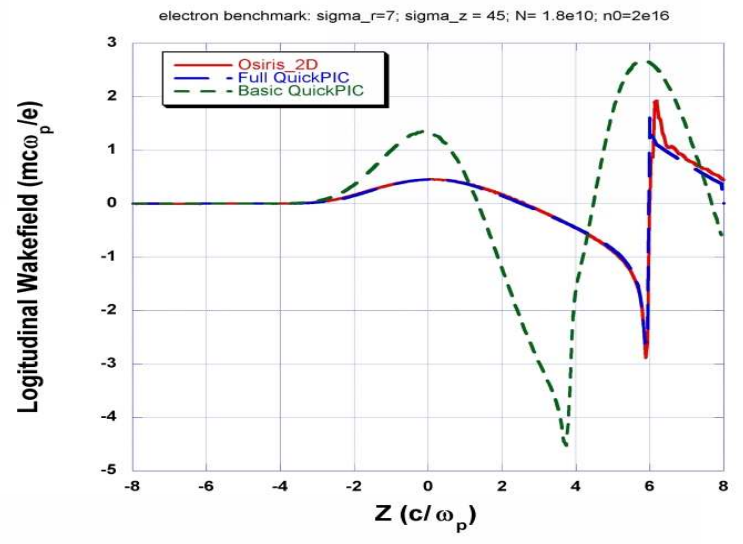

FIGURE 4. Comparison of the longitudinal electric field of the wake of an electron beam as computed by the basic QuickPIC algorithm, the full QuickPIC algorithm, and a full 2D EM-PIC simulation by OSIRIS.

\section{COMPUTATION DEVELOPMENT NEEDS}

While computation has been so important in recent breakthroughs in advanced acceleration, it can make further contributions with additional development. Clearly, the work on development of reduced models should continue, as it is the only hope for modeling some systems for a long time to come, given that with conventional methods, computational power would have to increase by several orders of magnitude. Additionally, the implementation of ionization physics needs to continue. The further development of ionization packages is also needed, with more types of ionization (impact by multiple species, more species of impact) covered. (Cline also noted the need for work on elastic scattering in advanced accelerator concepts.) In addition, the working group identified two other areas requiring more computational development. The interaction of laser pulses with solid density matter may provide a ion source. However, such modeling is difficult because of the fact that the plasma frequency is greater than the laser frequency. Increasingly dense and cold beams are being produced for multiple applications. The modeling of these systems is difficult due to the presence of a large variation of spatial scales.

\section{Laser-solid interactions}

The interaction of intense laser pulses with solid-density matter may provide a source of ions. Studies of this process were presented by Bowes [5], who used a radiation transport approach, and DeSilva [6], who used a particle-in-cell approach. The radiation transport approach does not do well at modeling the high-energy electrons that are produced, and it can fail to capture certain collective effects. On the other hand, the PIC approach has difficulty with high-density materials. Hence, approaches that could 
capture the full range of physics are needed.

Even within the PIC approach there appears room for improvement. The basic PIC approach must resolve the Debye length when the plasma is warm. The latter is needed to eliminate "grid heating." Debye length resolution requires $\Delta x<\lambda_{e}=v_{e} / \omega_{e}$, where $v_{e}$ is the electron thermal velocity. The Courant condition requires $\Delta t<\Delta x / c$. Taken together, these inequalities lead to the requirement, $\omega_{e} \Delta t<v_{e} / c$, i.e., the plasma frequency must be over-resolved by the ratio of the electron thermal speed to the speed of light. This can increase the computational requirement by several orders of magnitude from the requirement of simply resolving the electron plasma frequency. Moreover, even resolving the plasma frequency may be excessive, as the phenomena of interest appear to be ion-acoustic shocks, which have frequency much less than the plasma frequency. New modeling methods that can remove these unneeded scales from the problem are needed.

\section{Cold beams and multiscales}

As mentioned above, in his plenary talk Lewellen pointed to the need for codes that account accurately for the evolving hierarchies of spatial and temporal scales. A talk by Fubiani in our working group on space-charge effects in ultrashort bunches served to illustrate and emphasize these points. In our working group Lewellen also gave a presentation on challenges in modeling ultrahigh-brightness beams. One application of such a gun is Ultrafast Electron Diffraction (UED) for cross-disciplinary time-resolved studies such as ultrafast melting, changes in protein molecular structure during radiation, highresolution 3D reconstructions of carbon nanotubes, and chemical reactions. The beam requirements for UED are $\sim 10^{9}$ electrons $(\sim 160 \mathrm{pC})$ per bunch, $\sim 2$ ps bunch length with sub- $\mu \mathrm{m}$ emittance, and $5 \mathrm{MeV}$ kinetic energy. These are stringent requirements; no such gun presently exists. The design of such a gun requires accounting for a wide variation of scales; the beam volume is a tiny fraction of the gun-cavity volume. Ascertaining the detailed properties of the beam as it evolves through the gun requires a very fine mesh, whereas computing the cavity fields requires only a relatively much coarser mesh. In this sense, hierarchies of spatial and temporal scales pertain as a matter of principle not just to the beam itself (as is required to account accurately for space-charge effects when they are important), but also to the overall hardware configuration. Such challenges represent the frontier of injector design.

As another example of multiscale physics, Bohn presented a study of beam-halo production. Specifically, he showed how collective modes and colored noise conspire to produce beam halo with much larger amplitude than could be generated by either phenomenon separately. Collective modes are inherent to nonequilibrium beams with space charge. Colored noise arises from unavoidable machine transitions and/or errors that influence the internal space-charge force. In the model that Bohn employs, lowest-order radial eigenmodes calculated self-consistently for a direct-current, cylindrically symmetric, warm-fluid Kapchinskij-Vladimirskij equilibrium serve to model the collective modes. He showed that even with weak space charge, small-amplitude collective modes, and weak noise strength, a pronounced halo is seen to develop if these phenomena act 
on the beam over a sufficiently long time, such as in a synchrotron or storage ring. This finding points again to the importance of retaining the hierarchies of scale; collisionless dynamics due to space-charge fluctuations in a nonequilibrium beam, whatever their source may be, will likely be chaotic and influence global beam properties over time scales that are short compared to the transit time of the beam through the accelerator.

\section{DESIRED FEATURES FOR CODE RELEASE}

In one of the working groups, we addressed the issue of ease of use. Many who develop computational applications are primarily interested in pursuing physics goals within their own research groups. The personnel in those groups are intimately familiar with the failings, quirks, and arcane methods for using those codes. However, there is much greater group of accelerator researchers who would like to use existing codes, but do not have the time to wade through the arcana. For enhanced productivity of these users, the following features are desired.

- Verification and validation

- Regression test suite

- Comparisons of computed results with analytic solutions

- Validations: comparisons with experimental results

- Documentation

- User manual

- Usage examples

- User assistance

- User discussion lists or professional support

- Graphical user interfaces: minimally for generating input files and monitoring runs, better would be for running the code from start to finish

- Code for sanity checking, e.g., numerical stability of parameter choices

In addition, the community should explore the following as potential new features for codes.

- Importing experimental data

- Generic parsers for importing problem descriptions

- Interaction with CAD packages

- Standard data formats. One proposed example is a photoinjector markup language.

- Web invocability so that users need not obtain and build the code locally. Dimitrov noted a prototype, OopicWeb, that can be seen at www.txcorp.com.

\section{SUMMARY AND CONCLUSIONS}

Computational accelerator physics is alive and well. Its health is evident in its contributions of new ideas for advanced acceleration concepts as well as for a better understand- 
ing of nonlinear dynamics in nonequilibrium beams. Verification studies show that our codes are correctly solving the model equations. Validation is showing that the models are correct. The life in computational accelerator physics is seen in the continual development of new methods for modeling accelerator physics, the existence of challenging problems awaiting solution, and the extension of this field to making predictions over the full range of spatial and temporal scales.

Beyond explicitly considering the questions of verification and validation, the working group also identified frontiers in computing. In the modeling of LWFA and PWFA concepts, one frontier is the development of reduced models, which do not require one to follow as many details of the physics while still faithfully representing the physics. Another frontier is the inclusion of more ionization effects, which are found to be dominating some PWFA experiments. Both of these areas could use additional work.

But additionally, the working group recognized that in many other areas modeling has only just begun, and it is desperately needed for continued progress in accelerator physics. Modeling cold beams, where space charge is critical, requires new and/or better tools for treating the wide range of spatial and temporal scales. Laser-solid interactions, which may provide ion injectors, are hard to model because the plasma frequency, being above the laser frequency, sets a very small temporal scale. The inclusion of multiscales, emission models, halo-wall interaction, etc., and their integration into modeling codes needs serious work.

Finally, a need was expressed for more formal code releases and features. For codes to be more useful to a wider range of scientists, they need documentation, graphical user interfaces, and CAD input. To aid in ease of use, a web model for code invocation was proposed.

\section{ACKNOWLEDGMENTS}

JRC was supported by U.S. Department of Energy grants DE-FG03-95ER40926, DE-FG02-01ER41178, DE-FG02-03ER83857, and DE-AC03-76SF00098 and NSF grant 0113907 . CLB was supported by U.S. Department of Energy grant DE-FG0204ER41323. This research used resources of the National Energy Research Scientific Computing Center, which is supported by the Office of Science of the U.S. Department of Energy.

\section{REFERENCES}

1. R. Fonseca, Standard Problems in Advanced Acceleration, this conference (2004).

2. M. S. Hur, Averaged-PIC Simulation of Laser-Plasma Interaction: Pulse Amplification by Raman Backscattering and Wake Field, this conference (2004).

3. B. A. Shadwick: Thermal Effects in Intense Laser-Plasma Interactions, this conference (2004).

4. J. A. Cooley et al, Progress in Quasistatic Modeling of Plasma-Based Accelerators: QuickPIC, this conference (2004).

5. Bowes, Ultrafast 2-D Radiative Transport in a Micron-Scale Aluminum Plasma Excited at Relativistic Intensity, this conference (2004).

6. De Silva, PIC Modeling of Overdense Interactions, this conference (2004). 
7. C. L. Bohn, Collective Modes and Colored Noise as Beam-Halo Amplifiers, this conference (2004).

8. G. Fubiani, Studies of Space Charge Effects in Ultrashort Electron Bunches, this conference (2004).

9. X. Chang, Compensation for Bunch Emittance in a Magnetization and Space-Charge Dominated Beam, this conference (2004).

10. D. A. Dimitrov, Particle-in-Cell Simulations of Intense Laser Pulses Coupling into Plasma Channels, this conference (2004).

11. J. Cooley: Broad-Energy Electron Beam Injection and Loading in Laser Wakefield Accelerators, this conference (2004).

12. A. Reitsma, Laser Wakefield Acceleration - A Fully Self-Consistent Analysis, this conference (2004).

13. H. Milchberg, Hydrodynamical Evolution of Plasma Channels, this conference (2004).

14. J. Rosenzweig, Energy Loss of a High Charge Bunched Electron Beam in Plasma: Simulations, Scaling, and Accelerating Wake-fields, this conference (2004).

15. Bruhwiler, Simulation of Ionization Effects for High- Density Positron Drivers in future Plasma Wakefield Experiments, this conference (2004).

16. D. A. Dimitrov, The IONPACK Library of Ionization Algorithms for PIC Codes, this conference (2004).

17. A. Kesar, Time and Frequency Domain Models for Smith-Purcell Radiation from a Two Dimensional Charge Moving Above a Short Grating, this conference (2004).

18. D. Cline, A Muon Cooling Ring with Lithium Lenses, this conference (2004).

19. J. Lewellen, High-Brightness Injector Modeling, this conference (2004).

20. W. Mori, Advances in Simulation Capability, this conference (2004).

21. K. Mima, PIC Simulation and Experimental Research on High Energy Particle Generation and Their Applications, this conference (2004).

22. R. Kishek, Modeling of Halos and Intense Beams, this conference (2004).

23. J. Lewellen, Modeling Injectors, this conference (2004).

24. R.A.Fonseca, L.O.Silva, R.G.Hemker, F.S.Tsung, V.K.Decyk, W.Lu, C.Ren, W.B.Mori, S.Deng, S.Lee, T.Katsouleas, and J.C.Adam: OSIRIS: A Three-Dimensional, Fully Relativistic Particle in Cell Code for Modeling Plasma Based Accelerators. In P.M.A. Sloot et al., editors, ICCS 2002, LNCS 2331, pp. 342-351, 2002.

25. C. Nieter and J. R. Cary, "VORPAL: a versatile plasma simulation code", J. Comp. Phys. 196, 448472 (2004).

26. E. Thrane, et al., Photoinjector Production of a Flat Electron Beam, in Proc. 2002 Linac Conference, 310-312 (2002). 